\title{
FLORIDI: INFORMACIÓN Y FILOSOFÍA
}

\author{
FLORIDI: INFORMATION AND PHILOSOPHY
}

\author{
Ignacio Hernández Antón ${ }^{1}$ \\ Universidad de Sevilla (España)
}

Recibido: 11-12-2012

Aceptado: 23-01-2013

Resumen: En este trabajo se pretende profundizar en la Filosofía de la Información como disciplina académica y en particular en la visión que tiene Luciano Floridi sobre ella. Se estudia el alcance, la definición y los problemas abiertos que conforman el núcleo de dicha corriente y se dan ciertas orientaciones para comprender bien el concepto primitivo de información como noción omnipresente en la sociedad de las tecnologías de la información y la comunicación.

Palabras-clave: Floridi, información, filosofía.

\begin{abstract}
This contribution intends to deepen into the Philosophy of Information, in particular, into Luciano Floridi's stance. The scope, a definition, and some open problems that comprise this discipline core are studied. Some orientations to understand the primitive conception of information as a pervasive notion in the information society we live in is given.
\end{abstract}

Key-words: Floridi, information, philosophy.

\section{Objetivos}

Tradicionalmente es común entender la información por medio de su cuantificación, en particular, es muy popular la orientación estadística de Shannon [Shanon 1948]. Es esta aproximación de dan ciertas suposiciones de índole filosófica que quedan implícitas y que han etiquetado a la Teoría de la Información como algo solo tratable en la ciencia. El objetivo principal de este trabajo es reclamar el rol de la Filosofía en el tratamiento, conceptualización y modelado del concepto de información.

[1] (iha@us.es) Ignacio Hernández Antón es Investigador del Grupo de Lógica, Lenguaje e Información del Dpto. de Filosofía, Lógica y Filosofía de la Ciencia de la Universidad de Sevilla. Líneas de investigación: Filosofía y Teorías de la Información, Lógica Epistémica Dinámica, Epistemología Formal, Flujo de Información y Gestión del Conocimiento entre otras. 
Para ello se estudia la visión de uno de los promotores de esta línea filosófica de trabajo: Luciano Floridi. En este trabajo se analiza cómo se puede dar una noción filosófica de la información y cómo está planteado el panorama filosófico actual sobre el estudio de la información. Para ello se escoge la estructura de plantear primero qué significa hacer filosofía para seguir exponiendo qué significa hacer filosofía de la información y qué tipos de problemas está interesada en tratar ya que una buena manera de entender una rama filosófica es a través de las temáticas que trabaja.

\section{El alcance y la definición de la Filosofía de la Información}

Desde el punto de vista del planteamiento del alcance de una disciplina (en este caso la Filosofía de la Información) podemos entender que nos encontramos con dos tipos de problemas: aquellos propios de la Filosofía de la Información y otros problemas filosóficos que se replantean o traducen al lenguaje informacional y/o computacional. Muchos problemas filosóficos pueden ser tratados usando el potente arsenal conceptual y terminológico que ofrecen las Ciencias y Filosofía de la Información y Computación [Floridi 2011]. Para delimitar los problemas básicos de la Filosofía de la Información, Floridi atiende al criterio del alcance o temática [Floridi 2009b, 2010, 2011] donde se distingue entre:

-Aquellos que tratan sobre el análisis del concepto de información y su dinámica (generación, transmisión o eliminación de la información).

-Los que se relacionan con nociones semánticas como las de verdad y de significado.

-Los que tratan de la relación con el concepto de (distintas formas de) inteligencia.

-Aquellos con carácter más ontológico que tratan sobre la relación información-naturaleza-ser.

-Los que se inmiscuyen en aspectos prácticos relacionados con los valores en el uso y la ética de la información.

Desde el punto de vista de Floridi [Floridi 2011], para que un área filosófica se convierta en un campo de investigación bien definido (es decir, pueda tener una definición estandarizada), aunque esta sea interdisciplinar, debe cumplir ciertas condiciones de autonomía:

-Ser capaz de apropiarse de manera clara, explícita y precisa de la interpretación de un campo de estudio " $x$ " y que se presente como una "Filosofía de $x$ ", por ejemplo, Filosofía del Conocimiento o Filosofía de la Naturaleza. 


\section{Floridi: información y filosofía}

-Que esta interpretación del campo de estudio se convierta en un atractor de nuevas investigaciones por parte de la comunidad académica de manera regular.

-Que, como atractor, supere pruebas de reducción de esta nueva interpretación a otras intepretaciones ya existentes, es decir, que no sea posible el reducir los principios, problemas y metodologías de esta nueva forma de conceptualizar a otras ya existentes.

-La nueva interpretación debe ser suficientemente rica para estar organizada en subcampos y por lo tanto promueva la especialicación del trabajo académico. Por ejemplo, podemos entender que la Filosofía de la Naturaleza puede tener subcampos de estudio como la Filosofía de la Física, la Biología o la Química.

Son ejemplos de estas formas de interpretar un área de estudio aquellas que dan respuesta a preguntas como "¿cuál es la naturaleza del conocimiento?” o “¿qué entendemos por significado?” que sientan las bases disparadoras de la Epistemología y de la Filosofía del Lenguaje, respectivamente. Al preguntarnos si una Filosofía de la Información es posible que cumpla con los cuatro requisitos anteriores Floridi anticipa una serie de aclaraciones preliminares.

Podemos decir que hay al menos dos formas de apropiarse e interpretar un campo de estudio [Floridi 2003, 2008, 2011]: fenomenológicamente o metateoricamente. La Filosofía del Lenguaje y la Epistemología son dos ejemplos de ser filosofías de dos fenómenos, a saber, las manifestaciones lingüísticas y constructos de significación y, por otro lado, las manifestaciones y constructos epistémicos. No tratan directamente con las teorías linguísticas o las ciencias cognitivas. La Filosofía de la Física y la Filosofía de las Ciencias Sociales constituyen un ejemplo de aproximaciones metateóricas: investigan los problemas y constructos que surgen de sistemas de conocimiento ya dados y organizados de alguna manera. Estos últimos son los que propiamente estudian fenómenos naturales o humanos. No es que toda la filosofía esté dividida en fenomenológica o metateórica; para Floridi, hay campos como la Filosofía de las Matemáticas o la Filosofía de la Lógica que combinan en sus investigaciones ambos enfoques. La Filosofía de la Lógica por ejemplo está segada metateóricamente en el sentido de que tiene cierta tendencia a concertrarse primariamente sobre los problemas conceptuales que surge de la Lógica entendida como una teoría matemático-formal de la inferencia válida, ver [Floridi 2010, 2011] y [Nepomuceno 2007] prestando menos atención a la Lógica como fenómeno del pensamiento, es decir, a la idea de racionalidad natural.

De la misma manera, la Filosofía de la Información está sesgada fenomenológicamente [Floridi 2003, 2008, 2011]: se preocupa principalmente de los fenómenos informacionales, del procesamiento de la información (computación

THÉMATA. Revista de Filosofía, Nº49 enero-junio (2014) pp.: 127-142 doi: 10.12795/themata.2014.i49.07 
de la información) y de aspectos de la sociedad de la información. Floridi define la Filosofía de la Información como:

"El área filosófica que trata:

-Investigaciones analíticas sobre la naturaleza conceptual y los principios básicos de la información, incluyendo su dinámica, utilización y las teorías científicas sobre ella; y

-La elaboración y aplicación de metodologías (conceptos, terminología, enfoques) teorético-informacionales y computacionales a problemas filosófico ya dados."

El primer punto de la definición establece que la Filosofía de la Información se apropia de una interpretación clara de un campo de estudio, a saber: la naturaleza de la información. Este es el sello distintivo de esta disciplina. Evidentemente, esto sólo sirve para demarcar a grandes rasgos un área de estudio, no para dar detalles sobre los problemas más concretos derivados de esa amplia temática (en la siguiente sección se desglosan algunas de las más importantes líneas de investigación que dan más concrección a los problemas asociados a la información).

La Filosofía de la Información ofrece una investigación crítica que no debe ser confundida con las teorías matemáticas o computacionales de la información y la comunicación (centradas en la cuantificación y en el análisis estadístico/probabilístico y algorítmico principalmente). Con el adjetivo "critica", Floridi apela a que esta disciplina está en conexión con otras áreas filosóficas pero que no es reducible a conceptos clave de dichas áreas como son los conceptos de conocimiento, significado, verdad o acción.

Por dinámica de la información Floridi [Floridi 2011] se refiere a: -La contrucción y modelado de escenarios informacionales: sus propiedades como sistema y la interacción de sus elementos. Estos escenarios se consideran ciertos agentes y un modelo de comunicación que intenta captar cómo la información es transportada entre esos agentes.

-Los ciclos de información: desde la generación de información, procesamiento y utilización para la toma de decisiones hasta eliminación de información.

-Computación de la información tanto en el sentido del procesamiento algorítmico mediante máquinas de Turing como en el sentido más amplio que cae bajo el concepto de procesamiento general de la información.

Aunque las Ciencias de la Computación ha atraído fuertemente la atención de los académicos en los últimos años, la Filosofía de la Información la considera como una de las formas de tratamiento (automático) de la infor- 
mación en el mismo sentido de que la Epistemología es la reflexión sobre el conocimiento no sólo sobre la percepción (como un tipo concreto de proceso cognoscitivo).

El segundo punto de la definición indica no sólo que la Filosofía de la Información es un nuevo campo por su temática, sino que también proporciona una metodología innovadora. Estas metodologías tienen una fuente común en las tecnologías de la información y de la comunicación (TIC's) y en la reflexión sobre la sociedad de la información y del conocimiento (SIC). Se heredan métodos, herramientas conceptuales y técnicas desarrolladas en las Teorías de la Información y en las Ciencias de la Computación para intentar aplicarlas a diferentes áreas filosóficas y con ello:

-Ampliar nuestra comprensión de las habilidades cognitivas y lingüísticas de humanos y animales y la posibilidad de generar formas artificiales de inteligencia.

-Analizar los procesos inferenciales y computacionales: desde la filosofía de la computación pasando por las lógicas epistémicas y dinámicas que capturan aspectos del flujo de información hasta las teorías de las situaciones.

-Explicar los principios organizacionales de la vida y de la agencia (teoría genérica sobre agentes inteligentes): filosofía de la vida artificial, implicaciones cibernéticas de los autómatas, sistemas de decisión racional basados en teorías de juegos donde la información se toma como valor económico.

-Idear nuevos enfoques para modelar sistemas físicos y conceptuales: ontologías formales, teorías de los sitemas de información, filosofía de la realidad virtual.

-Formular conceptos sobre la metodología de la ciencia y sobre el concocimiento científico: teoría de modelos para la filosofía de la ciencia, metodologías computacionales en filosofía de la ciencia.

-Investigar sobre problemas éticos, estéticos y antropológicos relacionados con la información: ética para la información en sistemas de información, teoría de la estética multimedia o la ciberfilosofía (filosofía del comportamiento e identidad humana en entornos virtuales).

Con esto se aprecia como se satisfacen los criterios de atracción investigadora. También indica que es necesario un nuevo marco que integre estas inquietudes pero que, a su vez, conserve la capacidad de especialización en diferentes sublíneas de investigación. Floridi subraya que la Filosofía de la Información posee un vocabulario (y por consiguiente un arsenal conceptual) muy poderoso, lo que significa que muchos de los problemas filosóficos pueden ser reformulados en términos informacionales. Esta potencia semántica es la 
gran ventaja de la Filosofía de la Información aunque se advierte que independientemente de que pueda existir una reformulación de problemas filosóficos en términos informacionales, esto no implica que necesariamente la naturaleza del problema sea genuinamente informacional.

\section{Problemas en Filosofía de la Información}

A continuación se enumeran una serie de subcampos (problemas abiertos a la Hilbert) de la Filosofía de la información con algunos detalles donde se puede apreciar cómo se desarrollan ciertas investigaciones filosóficas [Floridi 2008, 2009a, 2010, 2011].

\subsection{Sobre el concepto de información}

La información es un concepto elusivo, primitivo pero casi omnipresente. La definición de información puede ser abordada desde, al menos, tres perspectivas.

-La información como realidad (lo que es la información): como patrones de señales físicas independientes del significado y de la verdad.

-La información sobre la realidad (de qué habla el conenido informacional): posee contenido semántico y se puede calificar de tener carácter alético.

-La información para (actuar sobre/en) la realidad: podemos hablar de instrucciones, algoritmos, información genética para la codificación de aminoácidos, etc.

A continuación se presenta someramente algunos de los paradigmas que han tratado la información como realidad y para la realidad. Estos han supuesto diferentes puntos de partida para la Filosofía de la Información. La lista siguiente no pretende ser exhaustiva:

-La Teoría Matemática (Estadística) de la Información y de la Codificación [Shannon 1948] [Nepomuceno et al. 2011] define la información en términos de distribuciones de probabilidad de ocurrencia de un símbolo sobre un alfabeto de símbolos dado.

-La Teoría Algorítimica de la Información (complejidad de Kolmogovoff) [Chaitin] definen la información contenida en una cadena $x$ como el tamaño en bits del menor algoritmo para computar (o caracterizar computacionalmente) $x$. 
-El Enfoque Probabilista [Bar-Hillel \& Carnap] [Dretske 1983] trata la información en términos semánticos y los relaciona con el espacio de probabilidad y el principio de relación inversa entre la información que tiene $p$ y la probabilidad de ocurrencia de $p$.

-El Enfoque Modal trata la información en términos del espacio lógico de consistencia. La información portada por $p$ es el conjunto de mundos posibles excluidos por $p$.

-El Enfoque Sistémico [Barwise \& Seligman] [Perry 1986] [Israel \& Perry 1990] [Devlin 1997] define la información en términos de estados y consistencia: la información sirve para hacer un seguimiento de las transiciones entre estados de un sistema que maneja información.

-El Enfoque Inferencial trata la información en términos del espacio de inferencia [Nepomuceno 2007, Floridi 2011]. La información depende de la inferencia válida relativa a la teoría del agente o del estado epistémico.

-El Enfoque Semántico define la información en términos del espacio de datos. La información semántica está compuesta por datos bien formados y verdaderos.

Muchos de estos enfoques tienen en común un espacio informacional en el que la información es vista como la reducción del grado de incertidumbre o en términos del nivel de sorpresa que genera en el agente receptor [DEL 2007, van Benthem 2008, Floridi 2011]. En la Teoría Matemática de la Información, el enfoque consiste en considerar la información como un fenómeno físico y además solo desde un nivel de relación entre símbolos, es decir, principalmente sintáctico: aquí la fuente de información es una fuente generadora de símbolos que tienen una probabilidad de ocurrencia dada; no se interesa en la utilidad de la información portada por la señal, ni en la relevancia, el significado, la veracidad o la temática sobre lo que versa el mensaje, sino en el nivel de detalle y frecuencia de los datos entendidos como señales sobre algún tipo de alfabeto o vocabulario. Aquí el interés se centra más bien en la cantidad de datos transmitidos por la señal, no de información en sí.

Algo similar ocurre en la Teoría Algorítmica de la Información, con la cuantificación de la información en términos de probabilidad y de longitud en bits de algoritmos de generación de cadenas. Aquí se interpreta la información y su cuantificación en términos de los recursos computacionales necesarios para especificarla.

Los enfoques restantes, de alguna u otra forma, intentan aproximarse a la información desde un enfoque semántico, preguntándose más bien sobre qué es el contenido que posee la información; normalmente adoptan una actitud proposicional en el sentido de analizar el contenido semántico de mensajes como "Hay un gato encima de la mesa". 
Aquí Floridi se plantea ciertas cuestiones de índole filosófica como: ¿son los enfoques sintácticos (los matemáticos y algorítmicos) los que establecen las condiciones necesarias para una teoría semántica de la información?, ¿son el resto de los enfoques semánticos compatibles a la vez?, ¿hay algún tipo de orden o jerarquía entre ellos?, ¿hay algún enfoque que profundice también sobre la noción de dato?

\subsection{Sobre la dinámica de la información}

El flujo de la información ha sido y es el centro de parte del esfuerzo de los estudios lógicos y filosóficos sobre la transmisión de la información llevada acabo por ciertos datos acerca de algo en un sistema distribuido de agentes inteligentes que procesan fragmentos de información [van Benthem 2008, Floridi 2006, 2011]. Hay, sin embargo, ciertos aspectos que necesitan ser explorados con mayor profundidad, por ejemplo: ¿cómo es posible que algo pueda portar información? Esta pregunta trata sobre la naturaleza de los datos como vehículos de información, la cuál es un tema central en la semiótica (y por tanto no es ajeno a la Filosofía del Lenguaje), en Hermenéutica y en disciplinas formales como la Lógica de las Situaciones.

La Lógica de la Información [Floridi 2006] (donde se propone un operado modal, $I \varphi$ que captura la idea de que un agente esta informado de que $\varphi$ ), usa el arsemal,de la Lógica Epistémica la cual reposa sobre el análisis doxástico del concocimiento [DEL 2007, van Benthem 2008, Floridi 2011] (usando $K \varphi$ y $B \varphi$ como operadores modales para capturar respectivamente las ideas deque un agente conoce que $\varphi$ y un agente cree que $\varphi$ ). Una de las preocupaciones insistentes de Floridi es acerca de si la Lógica Epistémica pueda englobar y/o dar cuenta de la Lógica de la Información y de la Lógica de las Creencias [Floridi 2006, 2011] o es la Lógica de la Información la que es más primitiva.

\subsection{Hacia una teoría única de la información}

Podríamos caer en la tentación de buscar una reducción de todos los enfoques antes expuestos sobre la noción de información a un único marco conceptual que los compatibilice, en este caso buscaríamos un teoría unificada de la información y de su dinámica [Floridi 2008, 2010, 2011]. No hay que descartar la posible irreduccibilidad de unos enfoques a otros de tener que conformase con un abanico de opciones conceptuales y ver el contexto de uso de la noción de información donde unos funcionan mejor que otros. 


\subsection{Sobre como adquieren los datos su significado}

Desde el punto de vista semántico para Floridi, la información puede ser analizada en términos de datos bien formados, con significado y que además son verdaderos (teoría semántica fuerte de la información). Si suponemos que los datos son intuitivamente descritos como diferencias sin interpretar (señales o símbolos), nos podemos preguntar acerca de cómo capturan o llegan a tener significado.

Esta cuestión está también relacionada con el establecimiento de las diferencias entre la inteligencia natural y la inteligencia artificial, asi como con la pregunta desde la Filosofía de la Mente sobre la conceptualización de la realidad, a saber: ¿cómo se generan los conceptos sobre la realidad?. También, desde el punto de vista metafísico, con la semantización del Ser (lo que es de alguna manera se convierte en algo significativo). Una vez que los datos bien formados han adquirido significado, para terminar de conformar un fragmento de información nos debemos preguntar sobre cómo adquieren su valor de verdad. Esto nos da pie al siguiente apartado.

\subsection{El valor de verdad de los datos}

Si partimos de una noción de información basada en el concepto de dato, una vez que estos adquieren significación, las preguntas naturales serían ¿de qué manera adquieren el valor de verdad?, ¿se puede entender la verdad y el significado mediante un enfoque informacional o es la información la que necesita ser analizada en términos de teorías no informacionales de la verdad y el significado? A raíz de estas preguntas surgen otras subsidiarias como aquella sobre si puede la información explicar la verdad, es decir, ¿se puede generar una teoría informacional de la verdad? Otro problema consistiría en explorar la posibilidad de si podemos diseñar una teoría semantica para explicar el significado [Floridi 2011]. Dretske [Dretske 1983], desde la Epistemología, ofrece algunos avances sobre estos enfoques informacionales de la semántica. Básicamente, este problema de la relación información-significado plantea la cuestión de si se puede fundamentar el significado en una noción objetiva como la de información, independiente de la mente y del lenguaje.

McCarthy y Hayes [Floridi 2011] subrayaban que un programa de ordenador que sea capaz de actuar inteligentemente en el mundo deberá tener una representación del mundo en función de cuáles de sus inputs son interpretados. Diseñar un programa así requeriría una dedicación sobre la investigación de lo que sea el conocimiento y de cómo es obtenido. De esta manera, uno de los grandes problemas de la Filosofía se incrusta en la Inteligencia Artificial. La Inteligencia Artificial y las Ciencias Cognitivas estudian los agentes cognoscentes desde, al menos, dos puntos de vista: desde la visión del procesa-

THÉMATA. Revista de Filosofía, Nº 49 enero-junio (2014) pp.: 127-142 doi: 10.12795/themata.2014.i49.07 
miento de la información como sistemas que reciben, almacenan, recuperan, transforman, generan y transmiten información y, por otro lado, desde la perspectiva computacional como clases de procesos algorítmicos (simbólicos) que pueden ser ejecutados por una máquina de Turing.

Los conceptos informaciones son tan potentes que a un determinado nivel de abstracción de lo que se esté hablando parece que casi todo puede ser replanteado como un sistema de información, desde el cerebro hasta un empresa. Es como si casi cualquier proceso puede ser simulado informacionalmente.

\subsection{La cognición como una forma de procesamiento de la información}

Una de las cuestiones relacionadas con la naturaleza de la inteligencia, mas concretamente con unos de los aspectos claves en la inteligencia, a saber, la cognición, es la que se pregunta sobre si la cognición puede ser descrita exclusivamente en términos de procesamiento de la infomación. Esto lleva a Floridi [Floridi 2008, 2011] a plantear varias cuestiones subsidiarias sobre la inteligencia natural y sobre algunos problemas clásico en Filosofía que desglosamos a continuación.

\subsection{La inteligencia natural como procesamiento de la información y su posible implementación no biológica}

¿Qué modelo de información puede describir correctamente la inteligencia natural y a qué nivel de abstracción podemos entender esta posible descripción? Estas preguntas dejan abierta la posibilidad de que la inteligencia natural o sea una clase de procesamiento de datos o sea demasiado compleja para que puede ser simulada mediante ingeniería. Si no fuera posible simular o implementarla biológicamente, ¿qué lo impide?, ¿la consciencia, la creatividad, la libertad?, ¿es simplemente un problema de tamaño, de cantidad de detalles y de complejidad? Perece invitable tratar aspectos de Filosofía de la Mente, por ejemplo, Floridi [Floridi 2010, 2011] se cuestiona si alguna teoría informacional tendría algo que ver con la idea de la dualidad mente-cuerpo o si la idea de identidad personal puede ser entendida en vez de físico-materialmente en términos de espacio informacional (privado-público).

Se suele decir que una de las diferencias entre la inteligencia artificial y la natural es que la primera puede identificar y procesar solamente datos (patrones de diferencias o invarianza no interpretados) mientras que la segunda identifica y procesa principalmente contenido informacional (datos bien formados y con significado). Parece ser que la inteligencia natural está atrapada en una posición semántica.

Siendo algo más precisos, nosotros no conocemos conscientemente datos puros con significado, lo que entendemos por datos puros son datos de los 
que carecemos de una interpretación específica relevante, no necesariamente es que no tengan interpretación. Quizás sea posible la percepción de datos no interpretados en ciertas circunstancias pero no es lo usual. Es difícil entender ese fenómeno como algo regular en la experiencia consciente, especialmente, porque escasean las situaciones donde nos enfrentamos a datos aislados; normalmente tenemos un contexto que facilita la interpretación semántica y la atribución de significado a dichos datos.

Floridi [Floridi 2003, 2009a, 2011] subraya que de las computadoras se dice que son máquinas sintácticas donde el nivel semántico o es despreciable (no necesariamente no existente) o es simulado mediante procesos sintácticos considerados desde la inteligencia natural como una proto-semántica o una semántica no tan rica como la que experimentamos los humanos. Extremando posiciones, se dice que, hasta lo que alcanza nuestro conocimiento, somo los únicos dispositivos realmente semánticos y que la inteligencia artificial está limitada por los recursos sintácticos de los que disponen sus lenguajes de programación y la inteligencia natural está limitada por recursos semánticos para interpretar los datos.

No deja de ser interesante hacer notar que conceder un mínimo de capacidad semántica a una máquina computacional da pie a empezar a aceptar una concepción extensionalista de la información como algo que está ahí afuera en el mundo más que algo que está exclusivamente en la mente de un ser vivo.

\subsection{Epistemología con base informacional}

Si se parte del realismo en Filosofía del Conocimiento, donde la información captura de alguna manera la forma en el que el mundo es, podemos preguntarlos sobre cómo el conocimiento encapsula la verdad en el sentido de que encapsula información semántica verdadera. Si el conocimiento presupone información, ¿puede haber estados informacionales sin estados epistémicos?, ¿es posible que un agente tenga una creencia verdadera sobre $p$ pero que no haya sido informado de que $p$ ?

Si particularizamos en la Epistemología podemos preguntarnos si las ciencias se reducen a un modelado de la información disponible, es decir, podemos preguntarnos: ¿cómo construimos los modelos científicos de (ciertas parcelas de) la realidad?, ¿es, en último término, información de lo que están contenidos o diseñados los modelos científicos?, ¿es la ciencia una actividad social (multiagente) que diseña información mediante clases de modelos?

\subsection{Ontología de la información}

Para Floridi [Floridi 2010, 2011], casi todo el mundo estaría de acuerdo en que no hay información sin cierta representación de los datos. Esto se en- 
tiende materialmente, es decir, es impensable tener un soporte físico de la información, la información está, de alguna manera, físicamente implementada. Desde el punto de vista de la Ontología, se puede preguntar si la información es una categoría ontológica diferente de lo material y lo mental. Si la información constituye o no una categoría ontológica independiente; si no lo fuera, ¿a qué categoría ontológica se reduciría? Si es una categoría independiente, ¿cómo se relacionaría esta con lo físico y con lo mental? Si hay información en el mundo independiente de cualquier forma de vida capaz de extraerla y procesarla, ¿cómo la caracterizaríamos?, ¿qué clase de acceso e interacción tendría un agente con esa forma de información-en-el-mundo?

La localización ontológica de la información está relacionada con la pregunta de si puede haber información sin informador o de si la información es algo esencialmente parasitario de los procesos semánticos en la mente de un informador. Para ilustrar esta cuestión, Floridi pone el ejemplo de la piedra Rosetta y se pregunta de si antes de su descubrimiento hubiera sido correcto pensar que los geroglíficos portaban información, incluso si su semántica no quedaba al alcance de ningún intérprete (vivo). Podemos preguntarnos si la información no está ni aquí, en la inteligencia, ni allí, en la naturaleza, sino que es una especie de interfase entre el mundo y sus habitantes.

En puntos más extremos de cuestionamiento Floridi [Floridi 2009b, 2011] trae a colación de posturas paninformacionales sobre la realidad: ¿es el universo en sí mismo esencialmente información y los procesos naturales como la causación son casos especiales de dinámicas informacionales? Si la naturaleza fuera información, ¿podría ayudar esto a explicar cómo la vida (mediante la información genética) emerge de la materia y luego la inteligencia (como procesador sofisticado de información) emerge de la vida?

\subsection{0. Ética e información}

Parece que lo último con que relacionaríamos con la información tal y como hemos venido hablando en este trabajo es con las acciones morales [Floridi 2008, 2011]. Si entendemos la información como un recurso para las evaluaciones y acciones morales de un agente, nos encontramos con un, casi inevitable, componente epistémico e informacional. Si un agente racional toma decisiones racionales se espera que lo haga usando la mejor información que posea. Floridi [Floridi 2006] recuerda que, en la Lógica Epistémica, que un agente sepa algo, $K \varphi$, se podría parafrasear como acorde con la mejor información que al agente tiene, entonces para ese agente $\varphi$. En principio, se puede suponer que el agente para tomar las mejores o más racionales decisiones (y por lo tanto ser susceptiblre de ser evaluadas moralmente) perseguirá obtener la mayor cantidad de información posible sobre la realidad, el curso de acción y sus consecuencias. 
Socrates pensaría (evidentemente no en estos términos) que un agente moral racional está de forma natural interesado en ganar la mayor cantidad de información pertienente que las circunstancias requieran puesto que un agente bien informado es mucho más probable que haga lo más conveniente moralmente; de hecho, el intelectualismo ético analiza la maldad de las acciones como resultado de un déficit de información. La responsabilidad moral de un agente racional tiende a ser proporcional al grado de información que posee. A menos información tendemos a asociar menor responsabilidad. No es lo mismo la sentencia de un hominicidio donde el asesino conoce (y por tanto tiene información) patente sobre las consecuencias de sus actos que si se prueba el desconocimiento (falta de consciencia sobre la información) de dichas consecuencias, por ejemplo, en el caso de un homicidio por negligencia. Como nota anecdótica, si uno se posiciona en la perspectiva de alguna religión como el cristianismo, la componente epistémica e informacional y la responsabilidad (en el caso del cristianismo asociada al perdón divino) no son claramente independientes: "Padre, perdónalos porque no saben (no tienen la mejor información sobre) lo que hacen" (Lucas 23:34).

Parece que un agente moral necesita información, probablemente mucha para operar correctamente. Pero incluso desde la perspectiva de entender la información como un recurso moral para la toma de decisiones hay que tener cierto cuidado con la idea de que cuanto más información mejor. Hay situaciones donde la carencia de información hace a un agente conseguir objetivos morales más deseables como, por ejemplo, en aquellas situaciones donde se pone en juego los derechos a la privacidad y al anonimato o en situaciones donde se pretende que se evalúen sin sesgo ciertas circunstancias para proceder a jucios imparciales. Para Floridi, nos podemos preguntar si estar informado de todo es siempre una situación deseable. Es, por tanto, la ética de la información la que trata temas como la disponibilidad, accesibilidad y confiabilidad de la información para la toma de decisiones.

No sólo un agente racional que toma decisiones es entendido como un consumidor de recursos informaciones, los agentes que toman decisiones son asimismo productores de información susceptibles de ser evaluadas moralemente. Ejemplos donde la producción de la información y la ética convergen son la publicidad, la propaganda política, la desinformación intecionada de masas, las calumnias y las mentiras.

Hay un tercer registro moral de información, esta vez atendiendo a cómo afectan las acciones y evaluaciones morales de un agente al entorno informaciones. Floridi pone el ejemplo de si un agente viola la privacidad o confidencialidad de la información de otro no es sólo un problema de entender la información como recurso (input) al que ha accedido ilícitamente sino que lo que también está en jego es la reflexion moral sobre el acceso no autorizado a cierta infromación sensible, independientemente del uso o maluso que se 
puede hacer luego con dicha información. Situaciones como el hacking, la piratería, la propiedad intelectual, el software libre, la libertad de experesión, las filtraciones periodísticas o la censura rodean este tipo de análisis moral de la información.

\section{Conclusiones}

Las tecnologías de la información y la comunicación, que crean, gestionan y utilizan la información y los recursos computacionales para aspectos de vital importancia hoy en día, no sólo influyen en nuestra compresión del mundo y en nuestra interacción con él, también influyen nuestra propia identidad y autoevaluación. Los humanos no somo entidades independientes, estamos conectados informacionalmente, Floridi utiliza el término inforgs, compartimos algo con agentes biológicos pero también con los artefactos de la ingeniería; ¿qué compartimos? un entorno que está compuesto por información, Floridi usa el término infoesfera. Las tecnologías de la información y de la comunicación no sólo son procesos de manipulación ingenieril del mundo, sino que llegan hasta producir procesos de re-ontologización del mismo.

Los objetos y los procesos están siendo desfisicalizados [Floridi 2010], se hacen cada vez más independientes del soporte físico (piénsese en un archivo de audio o en un trámite con una administración pública). Esto se hace en base a la reformulación informacional de problemas y soluciones y es por ello que la Filosofía de la Información está hoy en día dentro de los asuntos calientes del mundo académico. Con este trabajo lo que se ha pretendido subrayar es, en pocas palabras, que desgranar lo que la información sea y como funciona es una de las laberínticas tareas que tiene por delante la Filosofía. 


\section{Referencias bibliográficas:}

[Bar-Hillel \& Carnap] Yehoshua Bar-Hillel y Rudolf Carnap, 'Semantic Information'. The British Journal for the Philosophy of Science, Vol. 4. No. 14, 1953, 147157.

[Barwise \& Seligman] Barwise, J., \& Seligman, J. Information Flow: The Logic of Distributed Systems. Cambridge: Cambridge University Press, 1997.

[Chaitin] G.J. Chaitin. Algorithmic Information Theory. Cambridge Tracts in Theoretical Computer Science, 1987.

[DEL 2007] Hans van Ditmarsch, Wiebe van der Hoek, and Barteld Kooi. 2007. Dynamic Epistemic Logic (1st ed.). Springer Publishing Company, Incorporated.

[Devlin 1991] Devlin, K. J. (1991). Logic and Information. Cambridge: Cambridge University Press.

[Dretske1983] FredI.Dretske.Knowledge\&theFlowofInformation.BradfordBooks MIT press, 1983.

[Floridi 2003] Floridi, L. (ed.): Information. En The Blackwell Guide to the Philosophy of Computing and Information, pp. 40-61. Blackwell, Oxford, 2003. [Floridi 2006] Floridi, L. The logic of being informed, Logique et Analyse, 2006, 49.196, 433-460.

[Floridi 2008] Floridi, L. Trends in the Philosophy of Information. En Handbook of Philosophy of Information. Pieter Adriaans (Editor), Johan F.A.K. van Benthem (Editor), Dov M. Gabbay (Series Editor), Paul Thagard (Series Editor), John Woods (Series Editor). Elsevier 2008.

[Floridi 2009a] Floridi, L. The information Society and Its Philosophy, The Information Society, 2009, 25.3, 153-158.

[Floridi 2009b] Floridi,L. Philosophical Conceptions of Information, Lecture Notes in Computer Science, 2009, 5363, 13-53.

[Floridi 2010] Floridi, L. Information: a very short introduction. Oxford University Press, 2010.

[Floridi 2011] Floridi, L. What is the Philosophy of Information. En Philosophy of Information. Oxford University Press, 2011.

[Israel \& Perry 1990] Israel, D., \& Perry, J. What Is Information? In P. P. Hanson (Ed.), Information, Language, and Cognition (pp. 1-28). Vancouver: University of British Columbia Press, 1990.

[Nepomuceno 2007] Nepomuceno, A. Information and Logic. En Current Topics in Logic and Analytic Philosophy. Universidad de Santiago de Compostela publicaciones, 2007.

[Nepomuceno et al. 2011] Nepomuceno, Quesada Salguero (editores). Información: tratamiento y representación. Universidad de Sevilla publicaciones 2011. [Perry 1986] Perry, J. From Worlds to Situations. Journal of Philosophical Logic, 15(1), 83-107; 1986.

THÉMATA. Revista de Filosofía, Nº 49 enero-junio (2014) pp.: 127-142 doi: 10.12795/themata.2014.i49.07 
[RaK 1995] Fagin, Halpern, Moses, Vardi. Reasoning About Knowledge. MIT Press, 1995.

[Shanon 1948] Shannon, C. (1948). The Mathematical Theory of Communication. Bell Systems Technical Journal.

[van Benthem 2008] van Benthem, J. Information is what Information does. En Handbook of Philosophy of Information. Pieter Adriaans (Editor), Johan F.A.K. van Benthem (Editor), Dov M. Gabbay (Series Editor), Paul Thagard (Series Editor), John Woods (Series Editor). Elsevier 2008. 